\title{
Evaluation of Palm Oil-Based Paracetamol Suppositories by Differential Scanning Calorimetry
}

\author{
Mohamed Ibrahim Noordin*, Chung Lip Yong, llyana Mofat, Zainorwan \\ Zainuddin, Aditya Arya and Shaik Nyamathulla \\ Department of Pharmacy, Faculty of Medicine, University of Malaya, Kuala Lumpur-50603, Malaysia
}

*For correspondence: Email: ibrahimn@um.edu.my, shaik_pharmacy@yahoo.com; Tel: +603 -7967 4909; Fax: +603 -7967 4964

\begin{abstract}
Purpose: To use differential scanning calorimetry (DSC) to predict the liquefaction time and dissolution profile of some commercial paracetamol suppositories as well as paracetamol suppositories formulated with palm oil base.

Methods: The suppository base was prepared by mixing hydrogenated palm oil and palm kernel stearin in a ratio of 8:2. Three commercial paracetamol suppositories ( $A, B$, and $C)$ were purchased, while product $D$, paracetamol suppository was formulated with suppository base using the double casting method. In-vitro drug release from suppositories was determined using DSC and the effect of liquefaction on drug release was studied. The relationship between suppository melting and dissolution was analysed statistically.

Results: The melting temperature of products $A$ and $B$ were 41.0 and $40.0^{\circ} \mathrm{C}$ respectively, whereas those of products $C$ and $D$ were 35.9 and $33.1^{\circ} \mathrm{C}$, respectively. The liquefaction time of the suppositories were $61.4(A), 54.2(B), 9.0$ (C) and $7.4 \mathrm{~min}(D)$. Less than $40 \%$ paracetamol was released from products $A$ and $B$ in $2 h$, while products $C$ and $D$ showed good dissolution with $>95 \%$ drug release in $15 \mathrm{~min}$. Statistical analysis using Pearson's test indicates that the proportion of melt in the suppository base correlates well with drug release $(p<0.05)$.

Conclusion: Paracetamol release from palm oil suppositories is rapid and correlates with melting of the base. DSC can be used to predict drug release in paracetamol suppository formulations.
\end{abstract}

Keywords: Palm oil, Liquefaction time, Paracetamol, Suppositories, Thermal analysis.

Tropical Journal of Pharmaceutical Research is indexed by Science Citation Index (SciSearch), Scopus, International Pharmaceutical Abstract, Chemical Abstracts, Embase, Index Copernicus, EBSCO, African Index Medicus, JournalSeek, Journal Citation Reports/Science Edition, Directory of Open Access Journals (DOAJ), African Journal Online, Bioline International, Open-J-Gate and Pharmacy Abstracts

\section{INTRODUCTION}

Oral route of drug administration is the preferred choice in drug delivery. However, other routes of drug administration have proved to be equally good in some situations and sometimes is an unavoidable choice in drug therapy. The rectal route of drug administration is one such route normally opted for in geriatric and paediatric patients and in those cases where the oral route is not viable, especially in conditions such as nausea, vomiting, convulsion, difficulty in swallowing, gastric irritation, inactivation of drugs in the gastric environment and first-pass effect on drugs $[1,2]$. The rectal route has several limitations such as lower surface area, poor absorption, poor dissolution due to limited fluid content, inactivation by microbes and poor patient compliance. Nonetheless, in spite of these limitations, the rectal route still can be an effective route of administration.

Suppositories designed to deliver drugs to the rectum upon insertion will soften or melt at rectal 
temperature, releasing the active drug to the site of absorption. Usually, fatty bases with a melting point near rectal temperature are used in suppositories as vehicles. Fatty acid base suppositories melt and release the embedded drug from the suppository matrix into the mucous layer of the rectal lining and dissolve in the rectal fluid. Drug in the rectal fluid diffuses through the epithelium mucous layer of the rectal wall, usually by passive diffusion and enters the systemic circulation [3]. The rate of absorption depends on factors such as the rate at which drug release occurs from the suppository, concentration at the absorption site, excipient properties, solubilisation in the rectal fluids and the ability of the drug to cross the membrane barrier at the site of absorption [4].

Although there are multiple events during the absorption of drug in the rectum, the melting of suppository base will determine the release and absorption of drug to a significant extent. If the suppository does not melt readily to spread over the absorbing surface, the bioavailability of the embedded drug will be hindered. Hence, the melting temperature of a fatty suppository base is a limiting factor for drug absorption into the systemic circulation. Cocoa butter (theobroma oil) is often considered to be the ideal suppository base. But due to its polymorphic tendency, its use is limited. Palm products having melting characters identical to that of theobroma oil as well as good stability will be an excellent substitute suppository base.

Several in-vitro tests are described in pharmaceutical compendia to correlate in-vitro and in-vivo performance of dosage forms in order to assure the quality, purity and uniformity in the formulations. Unfortunately, many tests are limited to solid pharmaceutical dosage forms and there is no reliable, robust and rapid technique to predict the in-vivo performance of suppositories. Due to a lot of variability in suppository dosage forms, it is difficult to predict in-vivo drug release with merely a single test. Although there are different methods in the literature, the results have been erratic and case specific. Hence, there is need to develop a simple, quick, and robust method which can be used to predict drug release from suppositories.

To assess the quality of a suppository as a drug delivery system, quality tests such as liquefaction time, softening time, disintegration and dissolution have been prescribed in the literature $[5,6]$. In this study, an attempt was made to use DSC in determining the liquefaction time and dissolution profile of paracetamol suppositories in order to characterize the drug release from both test and commercial paracetamol suppositories. EXPERIMENTAL

\section{MATERIALS}

Paracetamol suppository $125 \mathrm{mg}$ (Product A) and paracetamol suppository $250 \mathrm{mg}$ (Product B) produced by the same manufacturer were purchased from a pharmacy in the Klang Valley, Kuala Lumpur, Malaysia. Paracetamol suppository $125 \mathrm{mg}$ (Product C) manufactured by a different manufacturer, was purchased from the same pharmacy. Paracetamol suppository 125 mg (Product D) was extemporaneously prepared according to the method described below. Hydrogenated palm kernel oil (Batch No. 0040933801), and hydrogenated palm kernel stearin (Batch No. 0091420002) were obtained from Cargill (M) Sdn Bhd, Kuala Lumpur, Malaysia. Stearic acid (Batch No. Tristar 149), glyceryl monostearate (Batch No. E01/096) and paracetamol (BP grade, Batch No. 020766) were donated by Hesego Industry (M) Sdn Bhd, Esterchem (M) Sdn. Bhd., and Raza Manufacturing Sdn Bhd, Kuala Lumpur, Malaysia, respectively. Other chemicals, which were of analytical grade, were purchased from Sigma-Aldrich Corp, St. Louis, USA and Fisher Scientific, Houston, USA.

\section{PREPARATION OF SUPPOSITORY BASE}

Palm kernel oil suppository base was prepared by mixing hydrogenated palm kernel oil and hydrogenated palm kernel stearin (8:2) using Erweka mixer (model AR 402) at $45{ }^{\circ} \mathrm{C}$ and paddle stirrer speed of $100 \mathrm{rpm}$, followed by the addition of stearic acid (5\% w/w) and glyceryl monostearate $(5 \% \mathrm{w} / \mathrm{w})$, according to our previously described method [7]. The blend was allowed to solidify at $25^{\circ} \mathrm{C}$ for one week and thereafter kept at $4{ }^{\circ} \mathrm{C}$ until use.

\section{PREPARATION OF PARACETAMOL SUPPOSITORIES}

Paracetamol suppositories with a dosage strength of $125 \mathrm{mg}$ each were prepared by melting the suppository base and incorporating sufficient paracetamol. In brief, the double casting method was adopted, and after the initial determination of the displacement value, the suppository base was melted at around $40{ }^{\circ} \mathrm{C}$. Subsequently, paracetamol was incorporated and gentle stirring was applied until a homogenous mixture of the base and drug was 
achieved. The molten mass was poured into stainless steel suppository moulds and the paracetamol suppositories were allowed to solidify at $25^{\circ} \mathrm{C}$ for 60 min before being released from the mould. A batch of 60 suppositories weighing about $1 \mathrm{~g}$ was prepared and used for the study immediately.

\section{THERMAL ANALYSIS}

The method adopted for the thermal study is similar to that reported in our previous work with few modifications [7]. Briefly, a differential scanning calorimeter (DSC 6, Perkin Elmer, USA) was connected to a chiller and a thermal analysis gas station (Perkin Elmer) to control the flow of the purge gas, nitrogen, at a flow rate of $20 \mathrm{ml} / \mathrm{min}$. The DSC pans used were $50 \mu \mathrm{l}$ closed aluminium pans with perforation. Indium and zinc (Perkin Elmer) were used to calibrate the DSC. Each sample weighing between 2.5 $4.0 \mathrm{mg}$ (UMT2 Microbalance, Mettler Toledo, Inc., USA) was scanned from 3.5 to $70^{\circ} \mathrm{C}$ at 5 $\mathrm{K} / \mathrm{min}$.

\section{Determination of liquefaction time}

Liquefaction test is usually done on suppositories to learn about the behaviour of suppositories in the rectal environment. The test is commonly referred to as Krowczynski's method and it measures the time needed for a suppository to liquefy in-vitro under conditions akin to the rectum [8]. The liquefaction time of the suppositories was determined by a custom made apparatus according to the specifications stated in the British Pharmacopoeia, 2000 [9]. The apparatus consists of three glass tubes immersed in water bath maintained at $37^{\circ} \mathrm{C}$. One end of the tube was closed and the other end was open for holding a glass rod. During the study $5 \mathrm{ml}$ of distilled water maintained at $37^{\circ} \mathrm{C}$ was poured into each of the tubes. Suppositories were inserted into the tubes with the tip facing downwards. Glass rod was placed above the suppositories, liquefaction time was recorded from the moment glass rod was placed on the suppository to the moment it just touches the tip of the glass tube.

\section{In-vitro dissolution study}

USP rotating basket method [10] was adopted for the dissolution study using Erweka dissolution apparatus (DT 70, Erweka GmbH, Germany). Five suppository samples were tested for each preparation. Each suppository was placed in the basket, lowered into the dissolution vessel containing $900 \mathrm{ml}$ of dissolution medium at $37^{\circ} \mathrm{C}$ and the basket rotated at $100 \mathrm{rpm}$. Samples (2 $\mathrm{ml}$ ) were withdrawn from the dissolution medium, filtered through $0.22 \mu \mathrm{m}$ filter and their absorbance measured at $244 \mathrm{~nm}$ using a spectrophotometer (1240 Mini UV, Shimadzu, Japan). The content of paracetamol was then determined from the absorbance using a standard curve for paracetamol at concentrations ranging from 15 to $300 \mu \mathrm{g} / \mathrm{ml}$. The accuracy of this method was assessed by spiking three known amounts of paracetamol $(75,150$ and 250 $\mu \mathrm{g} / \mathrm{ml} ; \mathrm{n}=5$ for each concentration) into the blank dissolution medium.

\section{Statistical analysis}

Statistical significance was performed using Prism 3 software (GraphPad Software, Inc., USA). The data were expressed as mean \pm standard deviation ( $S D, n=5)$, and analysed by Pearson's correlation test. Statistically significant difference was set at $p<0.05$.

\section{RESULTS}

Drug release from a suppository usually starts after melting of the suppository in the rectum and this is influenced by temperature, as well as the lipophilic and hydrophilic properties of the base, rectal fluids, surfactants and drug concentration at the absorption interface [11].

\section{Liquefaction time}

The results in Table 1 show that the melting point of suppository A and B is 41.0 and $40.0{ }^{\circ} \mathrm{C}$, respectively, and are higher than the body temperature $\left(37^{\circ} \mathrm{C}\right)$. Thus, products $A$ and $B$ required prolonged time and elevated temperature to achieve complete melting. Products $C$ and $D$ showed melting points of 35.9 and $33.1^{\circ} \mathrm{C}$, respectively, which were less than body temperature, and also exhibted short liquefaction time (Table 1).

\section{Thermal analysis}

The melt profiles of the suppositories, heat flow into the suppositories and subsequent phase transitions are depicted in the DSC thermograms shown in Fig 1.

The comparison of melting (\%) and drug release from the suppositories in 15 min is shown in Figure 2.

\section{In-vitro drug release studies}

Drug release from products $A$ and $B$ in 15 min was 1.2 and $6.4 \%$, respectively, while for $C$ and D, it was 95.6 and $99 \%$ (Table 2). 
Table 1: Melting characteristics of suppositories (mean $\pm S D, n=5$ )

\begin{tabular}{|c|c|c|c|c|c|}
\hline SP & $\begin{array}{l}\text { Liquefactio } \\
\text { n time (min) }\end{array}$ & $\begin{array}{l}\text { Melting } \\
\text { point }\left({ }^{\circ} \mathrm{C}\right)\end{array}$ & $\begin{array}{l}\text { Temperatur } \\
\text { e at } 100 \% \\
\text { melt }\left({ }^{\circ} \mathrm{C}\right)\end{array}$ & $\begin{array}{l}\text { Time interval } \\
\text { between } 0 \text { and } \\
100 \% \text { melt (min) }\end{array}$ & $\begin{array}{l}\text { Starting and end } \\
\text { melting points } \\
\text { (min) }\end{array}$ \\
\hline A & $61.4 \pm 1.7$ & $41.0 \pm 2.8$ & $63.4 \pm 10.8$ & $22.5 \pm 0.2$ & $12-22.5$ \\
\hline B & $54.2 \pm 1.7$ & $40.0 \pm 0.6$ & $46.1 \pm 1.7$ & $18.5 \pm 0.3$ & 3-18.5 \\
\hline C & $9.0 \pm 0.5$ & $35.9 \pm 1.7$ & $38.1 \pm 1.4$ & $7.1 \pm 0.5$ & $1-7.1$ \\
\hline D & $7.4 \pm 0.4$ & $33.1 \pm 1.4$ & $36.8 \pm 1.6$ & $7.0 \pm 0.2$ & $2-7$ \\
\hline
\end{tabular}

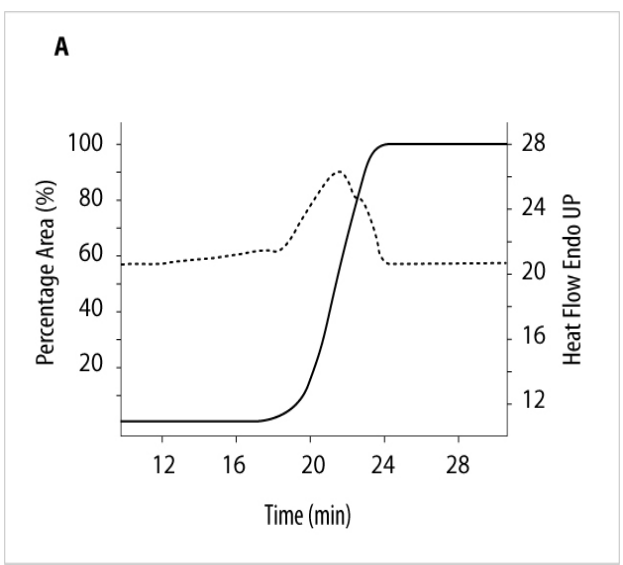

B

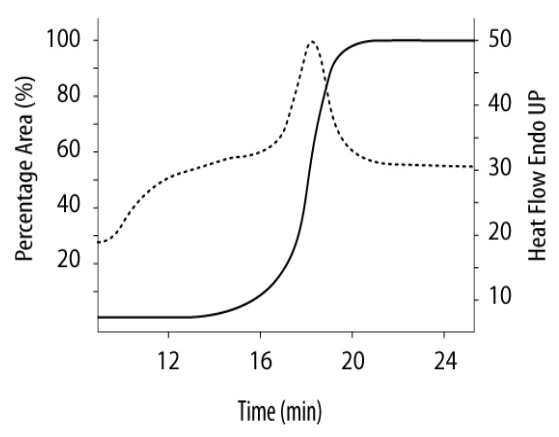

D

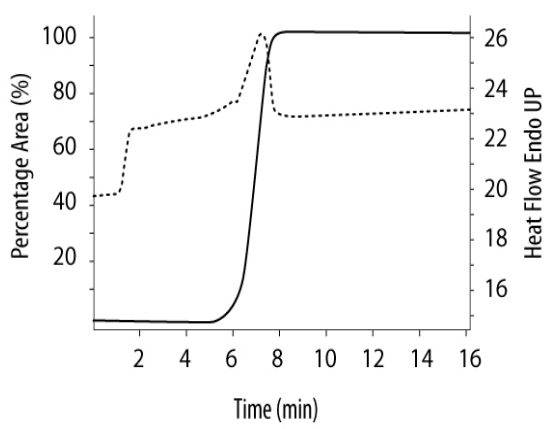

Fig. 1: Representative thermograms of suppositories A, B, C and D (denoted A, B, C and D. Respectively)

\section{DISCUSSION}

Among several factors, the melting characteristics of any suppository will determine its bioavailability and therapeutic efficacy to a significant level [12]. However, there are suppositories in which drug release occurs by slow dissolution instead of melting.

Modern thermal analysis has advanced in the last few decades and has a useful tool in drug development. Differential scanning calorimetry (DSC) is a powerful thermal analytical technique that has been extensively employed in the core areas of pharmaceutical development. It is usually used to determine drug purity, stability, content, melting point assessment, polymorphism, and compatibility between drug and excipients [11]. DSC measures the heat flow into or out of the sample and subsequent phase transitions under controlled environment over a wide range of temperature conditions programmed at a predetermined rate.

The thermograms of all the four suppositories have noticeable differences indicating the complexity of the composition of the suppository bases used by different manufacturer. Product $A$ showed a broad thermogram, attributed to complex fatty acids in the base with different thermal characters. In product A melting started at $12 \mathrm{~min}$ and ended at $22.5 \mathrm{~min}$ and had a peak melting temperature of $41^{\circ} \mathrm{C}$. Similarly product B showed $40^{\circ} \mathrm{C}$ as peak melting temperature with 

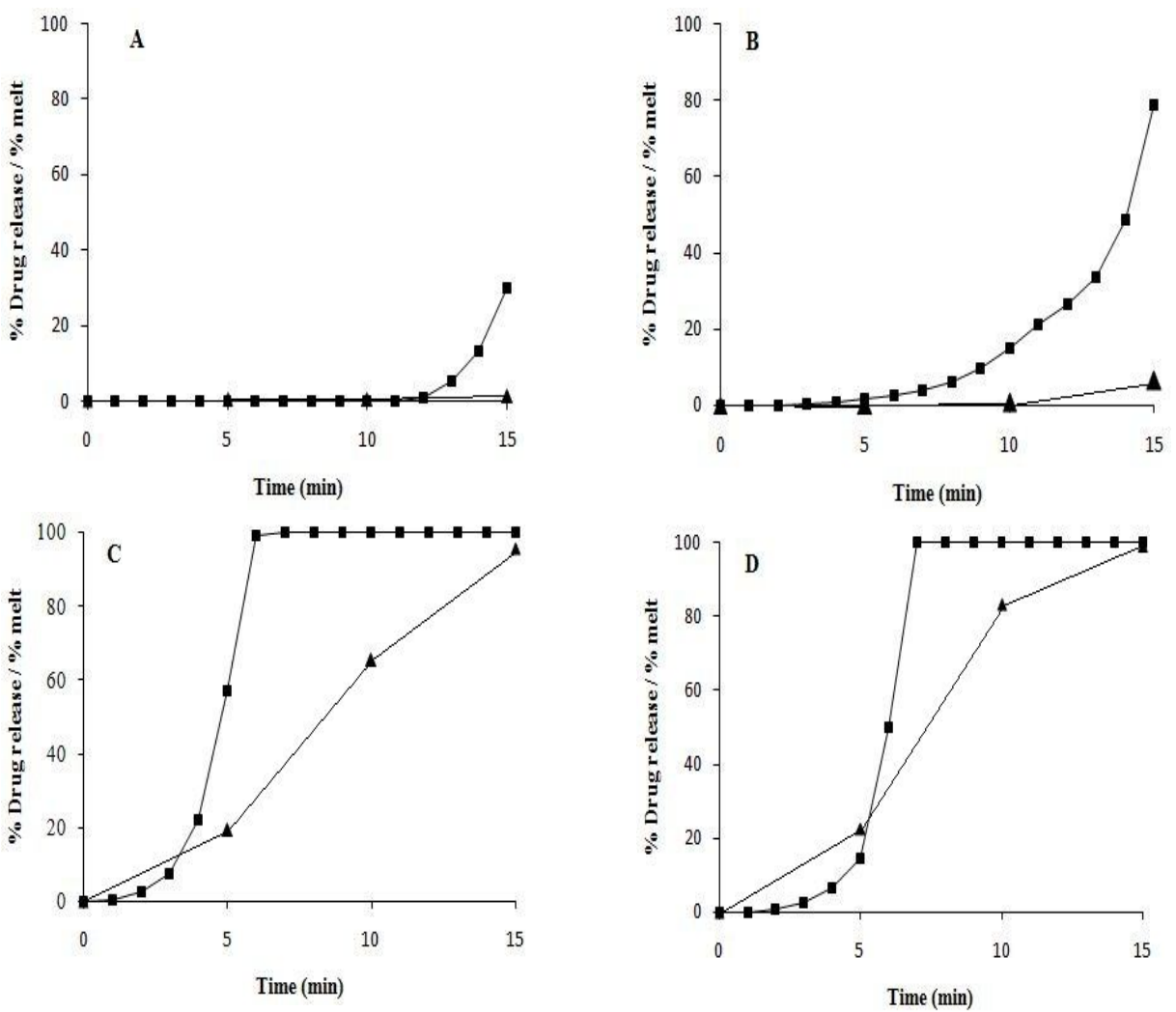

Fig. 2: Plots of paracetamol release suppositories $A, B, C$ and $D$, respectively

Table 2: Drug release from the suppositories (mean $\pm S D, n=5$ )

\begin{tabular}{|c|c|c|c|c|}
\hline \multirow{2}{*}{$\begin{array}{l}\text { Time } \\
\text { (min) }\end{array}$} & \multicolumn{4}{|c|}{ Drug release $(\%)$} \\
\hline & $\begin{array}{c}\text { Suppository } \\
A\end{array}$ & $\begin{array}{c}\text { Suppository } \\
\text { B }\end{array}$ & $\begin{array}{c}\text { Suppository } \\
\text { C }\end{array}$ & Suppository D \\
\hline 0 & 0 & 0 & 0 & 0 \\
\hline 5.0 & $0.2 \pm 0.0$ & $0.1 \pm 0.0$ & $19.2 \pm 0.8$ & $22.7 \pm 2.4$ \\
\hline 10.0 & $0.3 \pm 0.0$ & $0.7 \pm 0.1$ & $65.2 \pm 1.2$ & $83.2 \pm 3.7$ \\
\hline 15.0 & $1.2 \pm 0.2$ & $6.4 \pm 0.6$ & $95.6 \pm 0.9$ & $99.0 \pm 0.8$ \\
\hline 30.0 & $1.7 \pm 0.1$ & $12.7 \pm 0.9$ & $97.1 \pm 0.8$ & $99.9 \pm 0.5$ \\
\hline 60.0 & $6.6 \pm 1.3$ & $32.1 \pm 2.1$ & $97.4 \pm 1.0$ & $100.2 \pm 0.5$ \\
\hline 90.0 & $10.8 \pm 0.8$ & $34.9 \pm 0.4$ & $98.2 \pm 1.2$ & $100.8 \pm 1.5$ \\
\hline 120.0 & $14.6 \pm 1.0$ & $35.3 \pm 0.7$ & $98.6 \pm 2.0$ & $100.6 \pm 1.7$ \\
\hline
\end{tabular}

Statistical analysis revealed significant correlation between drug release and degree of suppository melt for all four formulations, with correlation coefficient $\left(r^{2}\right)$ values ranging from 0.7620 to 0.9877 and $p$ values of 0.0001 to 0.0280 . Furthermore, liquefaction time and the time taken to achieve complete melting were also correlated (Pearson's test, $r^{2}=0.9946$ and $p=0.0054$ ).

3 min as starting and 18.5 min as the end point.

Both these products were from the same manufacturer and probably might have used similar base. The thermogram of the product $B$ is sharper with narrow thermal range for the base. Due to batch to batch variation, dose difference (product $\mathrm{A}$ and $\mathrm{B}$ ), possible polymorphic forms, variable constituents of base or stability of base, the thermograms differ with each other. Usage of suppository bases like PEGs in product $A$ and $B$ which release the drug by dissolution rather than melting cannot be overruled.

In product $\mathrm{C}$ melting started at $1 \mathrm{~min}$ and ended at $7.1 \mathrm{~min}$. The peak melting temperature was $35.9{ }^{\circ} \mathrm{C}$. The thermogram of the product $\mathrm{C}$ resembles that of theobroma oil showing close polymorphic peaks. Theobroma oil has several polymorphic forms of which three forms are stable with different melting temperatures, 17, 22 and $33^{\circ} \mathrm{C}$. These polymorphic forms precipitate 
in the theobroma base upon overheating the base during suppository preparation. Probably due to the presence of these polymorphic forms the thermogram of product $C$ has shown polymorphic peaks. Product D started melting at $2 \mathrm{~min}$ and ended at $7 \mathrm{~min}$. Its thermogram was relatively sharper with peak melting temperature of $33.1^{\circ} \mathrm{C}$. Palm oil base has selective fatty acids as it was the composition of hydrogenated palm kernel oil and palm kernel stearin at 8:2 ratios, these selected pure components exhibited rapid, sharp melting characters in product $D$. Temperature required to melt $100 \%$ of the base also has considerable variation among the products with $63.4,46.1,38.1$ and $36.8^{\circ} \mathrm{C}$ for $\mathrm{A}$, $B, C, D$ respectively.

Phase transitions depend, to a large extent, on the physicochemical properties of the ingredients in the suppository, and can influence liquefaction time and dissolution. Products $A$ and $B$ showed high liquefaction time of 61.4 and $54.2 \mathrm{~min}$, respectively. On the other hand, the liquefaction time of products $C$ and $D$ was 9.0 and 7.4, respectively. As per the pharmacopoeial requirement liquefaction time should be less than $30 \mathrm{~min}$. These results suggest that products $\mathrm{C}$ and $D$ will melt rapidly after insertion into the rectum.

Products $A$ and $B$ failed to achieve complete drug release after $2 \mathrm{~h}$ while products $\mathrm{C}$ and $\mathrm{D}$ released $>95 \%$ of after the drug in $15 \mathrm{~min}$. Excipients can inhibit drug release from suppository matrice. The mechanism of drug release from the above suppositories might be influenced by the viscosity of the molten suppositories. The poor drug release from products $A$ and $B$ can be attributed to their unmelted or partially melted base. Whereas products $C$ and $D$ has higher drug release with complete or near complete melt. Thus in the present study molten base might have regulated the drug release due to changes in the viscosity $[12,13]$. The suppositories prepared with palm oil base (D) offered good melting and drug release properties and thus, palm oil base may be a good replacement for cocoa butter.

The results obtained showed a correlation between thermal analysis and in vitro drug release, and this is significant from quality control perspective of suppository dosage forms. United States Food and Drug Administration (USFDA) in their guidelines in 2003 [14] stresses the importance of in-vitro/in-vivo correlations for pharmaceutical dosage forms. The present study will suggests that DSC is a suitable alternative to conventional dissolution tests for the evaluation of in-vitro drug release from suppositories. Thus, unlike other pharmaceutical dosage forms, it may be possible to obtain a good correlation between in-vitro and in-vivo performance of suppositories using the tool of thermal analysis, [15,16]. A major advantage of DSC is that it is relatively simple, robust and rapid.

\section{CONCLUSION}

DSC would be particularly suitable for the evaluation of suppository formulations to predict dissolution rate in a short period. Although it cannot be used as a replacement for existing invitro dissolution studies, the method can serve as a supportive tool where quick assessment is required.

\section{REFERENCES}

1. Itoh S, Teraoka N, Matsuda T, Okamoto K, Takagi T, Oo C, Kao HD. Reciprocating dialysis method: periodic tapping improved in-vitro release/dissolution testing of suppositories. Eur. J. Pharm. Biopharm., 2006; 64: 393-398.

2. Janicki S, Sznitowska M, Zebrowska W, Gabiga H, Kupiec M. Evaluation of paracetamol suppositories by pharmacopoeial dissolution test-comments on methodology. Eur. J. Pharm. Biopharm. 2001; 52: 249-254.

3. De Blaey CJ, Tuckker TJ. Suppositories and Pessaries. In: Aulton ME, editor. The Science of the Dosage Form Design; International Student ed. UK: Churchill Livingstone, 1996; pp 412-420.

4. Realdon N, Ragazzi E, Zotto MD, Fini GD. Layered excipient suppositories: the possibility of modulating drug availability. Int. J. Pharm. 1997; 148: 155-163.

5. Gold M. Suppository Development and Production. In: Lieberman HA.; Rieger MM.; Banker GS., et al., editors. Pharmaceutical Dosage Forms: Dispersed System. New York: Marcel Dekker Inc, 1988; pp 533-565.

6. Victoria MM and David CJ. Thermal and rheological study of lipophilic ethosuximide suppositories. Eur. J. Pharm. Sci. 2003; 19: 123-128.

7. Noordin MI, Chung LY. A rapid microquantification method of paracetamol in suppositories using differential scanning calorimetry. Drug Dev. Ind. Pharm. 2004; 30: 925-930.

8. Giron G, Riva A, Steiger M. DSC as support for the development of suppositories. Thermochim. Acta. 1985; 85: 509-512.

9. British Pharmacopoeia. Softening time determination of lipophilic suppositories; British Pharmacopoeia Commission, HMSO: London, 2000; A300.

10. United States Pharmacopoeia.National Formulary, USP 23, Rockville, United States of America, 2000; pp 1791-1799.

11. Chicco D, Grabnar I, Skerjanec A, Vojnovic D, Maurich $V$, Realdon N, Ragazzi E, Belic $A$, Karba $R$ and Mrhar $A$. Correlation of in vitro and in vivo paracetamol availability from layered excipient suppositories. Int. J. Pharm. 1999; 189: 147-160.

12. Hanaee J, Javadzadeh $Y$, Taftachi $S$, Farid $D$, Nokhodchi $A$. The role of various surfactants on the release of salbutamol from suppositories. Farmaco. 2004; 59: 903-906.

13. Shen E, Kipper MJ, Dzaidul B, Lim MK, Narasimhan B. Mechanistic relationships between polymer 
microstructure and drug release kinetics in bioerodible polyanhydrides. J. Control Release. 2002; 82: 115-125.

14. Guidance for Industry. Extended release solid oral dosage forms: Development, evaluation and application of in vitro/in vivo correlations; Center for Drug Evaluation \& Research (CDER), Food and Drug Administration (FDA): United Sates of America, 2003. Available from: http://www.fda.gov /downloads/Drugs/Guidances/UCM070636.pdf.
15. Novoa GAG, Heinamaki J, Mirza S, Antikinen O, Colarte Al, Paz AS, Yliruusi J. Physical solid-state properties and dissolution of sustained release matrices of polyvinylacetate. Eur. J. Pharm. Biopharm. 2005; 59: 343-350.

16. Mura P, Adragna E, Rabasco AM, Moyano JR, PerezMartinez Jl, Arias MJ, Gines JM. Effects of the Host cavity size and the preparation method on the physicochemical properties of Ibuproxamcyclodextrin systems. Drug Dev. Ind. Pharm. 1999; 25: $279-287$. 